\title{
Role of MRI for Evaluation of Uterine Giant Myoma: Case Report
}

\author{
Trapuzzano Orazio ${ }^{1}$, Caputo Nicoletta ${ }^{1}$, Marchese Stefano ${ }^{1}$, Lacamera Ugo ${ }^{2}$, \\ Tamburrini Stefania ${ }^{3}$, Smiraglio Claudia ${ }^{1}$ \\ ${ }^{1}$ Department of Experimental and Clinical Medicine-Radiology, University of Catanzaro "Magna Graecia”, Catanzaro, Italy \\ ${ }^{2}$ Department of Medical and Surgery Sciences, Clinical Surgery Unit University of Catanzaro "Magna Graecia", Catanzaro, Italy \\ ${ }^{3}$ Departement of Radiology, Pellegrini Hospital, Naples, Italy
}

\section{Email address:}

orazio.trapuzzano@libero.it (T. Orazio)

\section{To cite this article:}

Trapuzzano Orazio, Caputo Nicoletta, Marchese Stefano, Lacamera Ugo, Tamburrini Stefania, Smiraglio Claudia. Role of MRI for Evaluation of Uterine Giant Myoma: Case Report. International Journal of Medical Imaging. Vol. 3, No. 3, 2015, pp. $59-62$.

doi: $10.11648 /$ j.ijmi.20150303.13

\begin{abstract}
Uterine leiomyomas are common, benign, smooth muscle tumors of the uterus, occurring in $20-30 \%$ of women over age 35. A giant uterine leiomyoma is a tumor whose weight exceeds the arbitrary limit of approximately $11.4 \mathrm{~kg}(25 \mathrm{lb})$. Commonly multiple, leiomyomas can be described submucosal, intramural, subserosal. Large leiomyomas can cause various types of degeneration. The interest of the case lies in the difficulty of placing a differential diagnosis for the morphological characteristics of the large mass, due to non-characteristic aspect to the US and the MDCT. So MRI allowed to establish the relationship of the tumor with the uterus through the recognition of the vascular peduncle that connects the leiomyoma with fundus of the uterus. The application of MRI in the large pelvic masses goes beyond lesion detection, allowing in this case the typing, which is confirmed by the surgical findings.
\end{abstract}

Keywords: Uterine Giant Myoma, MRI-Pelvis, Pelvic Masses

\section{Introduction}

Uterine leiomyomas are common, benign, smooth muscle tumors of the uterus, occurring in $20-30 \%$ of women over age 35 ; the prevalence increases during the reproductive age and decreases after menopause. Commonly multiple, leiomyomas can be described submucosal, intramural, subserosal; the latter may become pedunculated and simulate ovarian neoplasms. Large leiomyomas can cause various types of degeneration: hyaline or myxoid degeneration, calcification, cystic degeneration, and red degeneration (1-5). A giant uterine leiomyoma is a tumor whose weight exceeds the arbitrary limit of approximately $11.4 \mathrm{~kg}(25 \mathrm{lb})(6)$.

Ultrasound is usually the initial screening tool for myoma(7).

Multidetector computed tomography (MDCT) scan has also been used as tool to diagnose uterine fibroids. Now its role is very much limited to cases of fibroids with complications such as necrosis and malignant transformation (8).
MRI is very useful for characterizing these tumors, which usually show low signal intensity similar to that of smooth muscle on T2-weighted images, but the presence of atypical degenerated leiomyomas make it differential diagnosis difficult $(9,10)$.

\section{Case Report}

A 42-year-old woman was referred to our institute for a chronic constipation associated with pain and abdominal distension for about 9 months. On physical examination, an abdominopelvic mass was palpated (Figure 1). Initially, the patient was subjected to laboratory tests and tumor markers (CEA, CA 19.9, CA125, CA72-4, TPA and total HCG), which were essentially normal. Hemoglobin was $11.5 \mathrm{~g} / \mathrm{dl}$. Creatininemie was $0.8 \mathrm{ml} / \mathrm{dl}$, GFR (glomerular filtration rate) was $101 \mathrm{~mL} / \mathrm{min}$. 


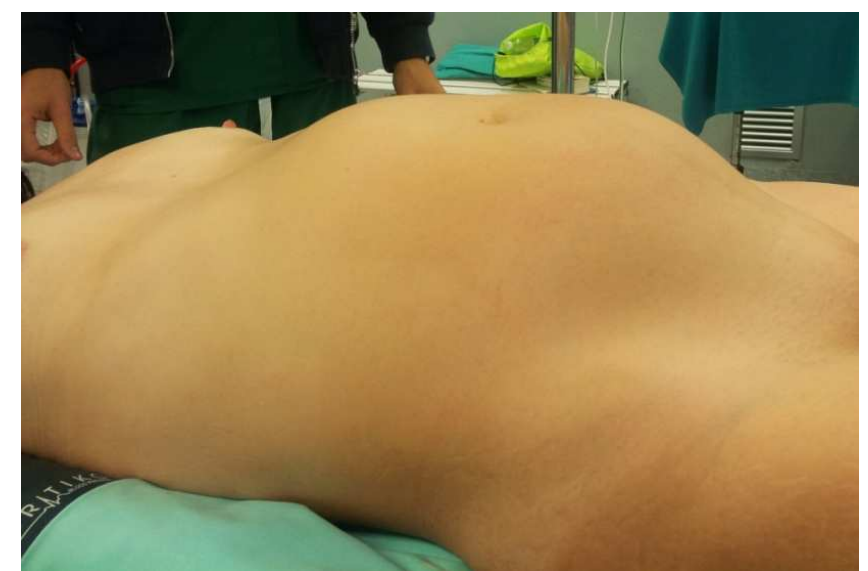

Figure 1. Preoperative evaluation: photograph of a 42 years-old woman with a distended abdomen.

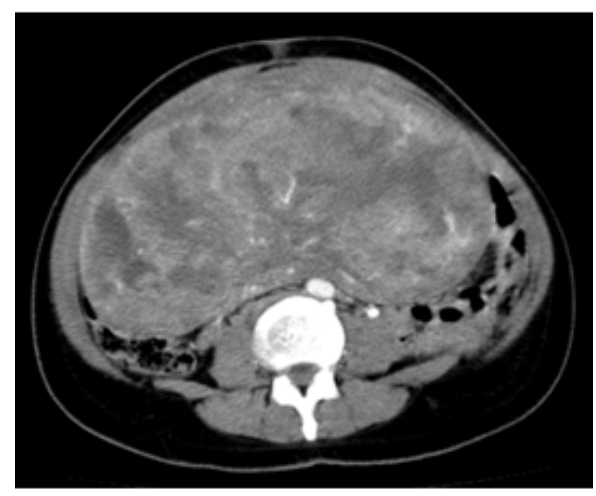

Figure 2a

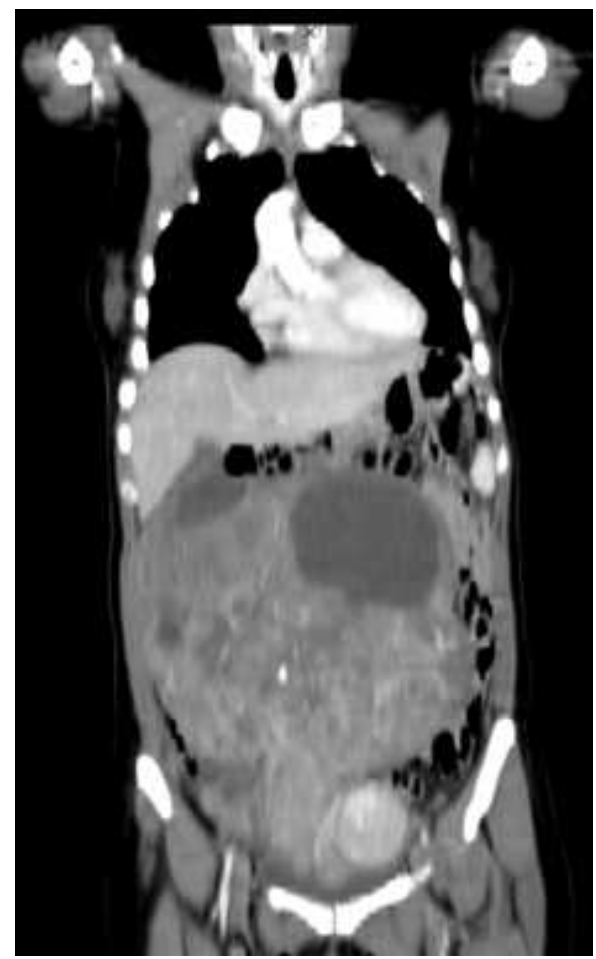

Figure $2 b$

Figure 2a-b. MDCT. Massive and widely heterogeneous solid lesion with necrosis phenomenonin context, massively occupying the abdomenand pelvis.
CTMD after contrast injection showed a large and widely heterogeneous solid mass with necrosis areas (Figure $2 \mathrm{a}-\mathrm{b}$ ). MRI revealed a large mass communicated with the uterus by fundic stalk. This mass was present in all abdominal cavity and pelvis (size: $25 \times 24 \times 11 \mathrm{~cm}$ ), with well-defined margins and mixed-signal, for the presence on T2-weighted images of hypointense solid areas and there is a hyperintense area reflecting cystic or necrotic changes with interior septa. The Large mass exerts significant local mass effect on adjacent structures (figure $3 \mathrm{a}-\mathrm{b}$ ). On T1 3D fat sat-weighted (Thrive) images after contrast injection showed intense, heterogeneous and progressive contrast enhancement of the large mass communicated with the uterus by fundic stalk (Figure 4). The first diagnostic hypothesis was a large mass communicated with the uterus by fundic stalk (giant leiomyoma uterine degenerate). The application of MRI in pelvic masses goes beyond lesion detection, allowing in this case the typing, which is confirmed by the surgical findings (Figure 5). The histological results of this mass was uterine giant leiomyoma.

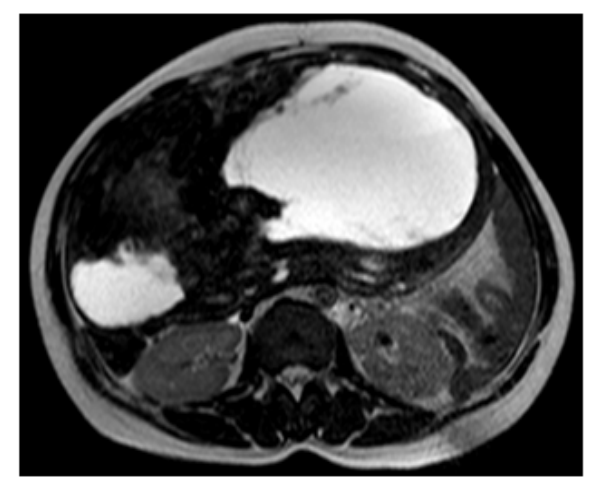

Figure 3a. Axial MRI T2wTSE

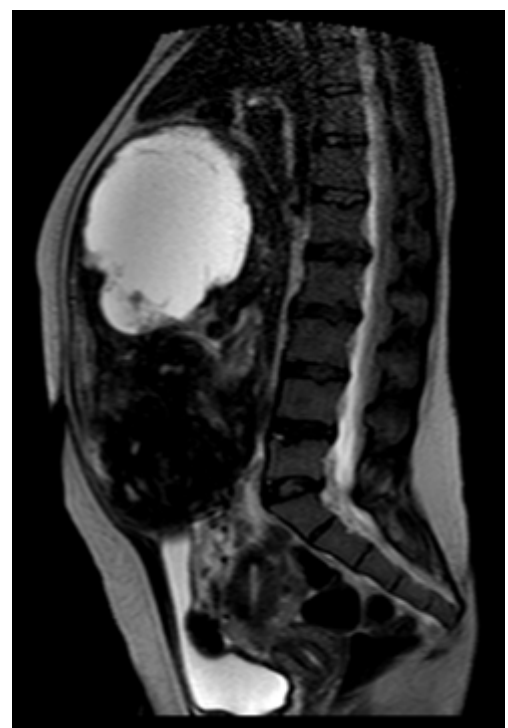

Figure 3b. Sagittal MRI T2wTSE

Figure 3 a- b. Showed large mass occupying most of the abdominopelvic cavity to intensity heterogeneous signal whit presence of cystic and necrotic degeneration. Sagital MRI showed the presence of large mass communicated with the uterus by fundic stalk. 


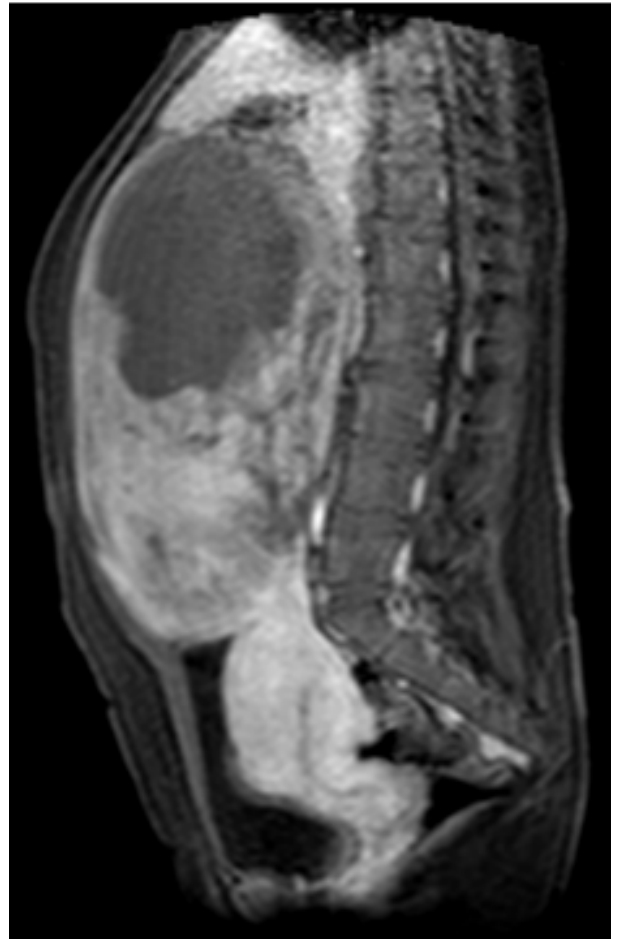

Figure 4. Sagittal MRI TI 3D FAT SAT after contrast injection showed intense, heterogeneous and progressive contrast enhancement of large mass communicated with the uterus by fundic stalk

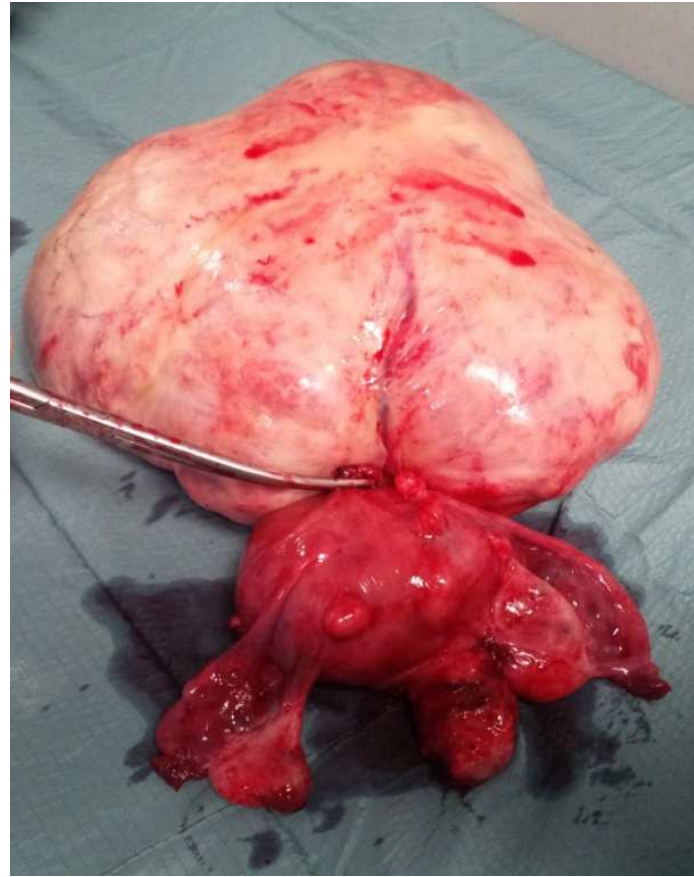

Figure 5. Leiomyoma giant, uterus with multiple smaller myoma and ovaries.

\section{Discussion}

Uterine leiomyomas are common, benign, smooth muscle tumors of the uterus, occurring in $20-30 \%$ of women over age 35; the prevalence increases during the reproductive age and decreases after menopause. Commonly multiple, leiomyomas can be described submucosal, intramural, subserosal; the latter may become pedunculated and simulate ovarian neoplasms (1-5).

Many masses in the female pelvis arise from the reproductive organs or may arise from the gastrointestinal system, urinary system, adjacent soft tissues, and metastases: so determines that the differential diagnosis of large masses female appears to be complex. With large tumors, it may not always be possible to determine the site of origin. Frequently, large masses in the female pelvis represent such commonly encountered entities as uterine fibroid tumor, dermoid tumor, ovarian cyst, and ovarian cancer. However, uncommon pelvic masses such as mesothelioma, adenocarcinoma, carcinosarcoma, laiomyosarcoma, and desmoid tumor may also be seen (9). They can become twisted and cause a kink obstructing blood vessels feeding the tumor that requires prompt surgery. Usually, uterine leiomyomas are diagnosed with ultrasound examination. Magnetic resonance imaging is used as a problem solving tool to find pathology uterine and adnexal (10).

\section{Conclusion}

The interest of this case lies in the difficulty of placing a differential diagnosis, since the symptoms presented by the patient was predominantly gastrointestinal because of compressive phenomena caused by the large size of the mass, in the absence of gynecological symptoms.

So MRI allowed to establish the relationship of the tumor with the uterus through the recognition of the vascular peduncle that connects the giant leiomyoma with fundus of the uterus. In fact, the MRI has provided an excellent anatomical map correctly directing the surgical approach.

\section{References}

[1] Fried AM, Kenney CM 3rd, Stigers KB, Kacki MH, Buckley SL. (1996) Benign pelvic masses: sonographic spectrum. Radio Graphics 16(2):321-34

[2] Fasih N, Prasad Shanbhogue AK, Macdonald DB, Fraser-Hill MA, Papadatos D, Kielar AZ, Doherty GP, Walsh C, McInnes M, Atri M. (2008) Leiomyomas beyond the Uterus: Unusual Locations, Rare Manifestations. Radio Graphics 28(7):1931-48

[3] Murase E, Siegelman ES, Outwater EK, Perez-Jaffe LA, Tureck RW. (1999) Uterineleiomyomas: Histopathological features, MR imaging findings, differential diagnosis, and treatment. Radio Graphics 19(5):1179-97

[4] Grapsa D, Smymiotis V, Hasiakos D, Kontogianni-Katsarou K, Kondi-Pafiti A. (2006) A giant uterine leiomyoma simulating an ovarian mass in a 16-year-old girl: a case report and review of the literature. Eur J Gynaecol Oncol. 27(3):294-6

[5] Dancz CE, Macdonald HR. (2008) Massive cystic degeneration of a pedunculated leiomyoma. Fertil Steril. 90(4):1180-1

[6] Nappi L, Matteo M, Giardina S, Rosenberg P, Indraccolo U, Greco P. (2008) Managment of uterine giant myoma. Arch Gynecol Obstet 278(1):61-3 
[7] Chopra S, Lev-Toaff AS, Ors F, Bergin D. (2006) Adenomyosis: Common and uncommon manifestations on sonography and magnetic resonance imaging. J Ultrasound Med. May;25(5):617-27

[8] Stadsvold JL, Molpus KL, Baker JJ, Michael K, Remmenga SW. (2005) Conservative management of a myxoid endometrial stromal sarcoma in a 16-year-old nulliparous woman. Gynecol Oncol. Oct; 99(1):243-5.
[9] Szklaruk J, Tamm EP, Choi H, Varavithya V. (2003) MR Imaging of Common and Uncommon Large Pelvic Masses. Radio Graphics 23(2): 403-424

[10] Sala E, Rockall A, Rangarajan D, Kubik-Huch RA.2010The role of dynamic contrast-enhanced and diffusion weighted magnetic resonance imaging in the female pelvis. Eur J Radiol. Dec; 76(3):367-85. 